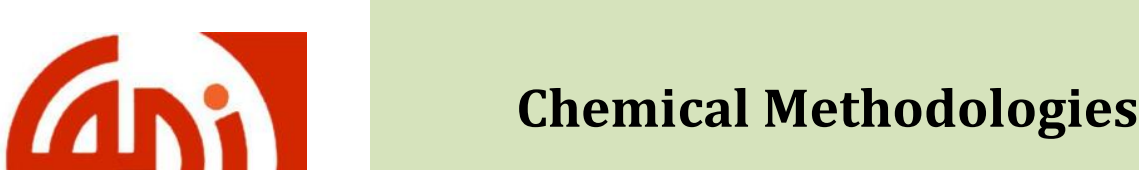 \\ Journal homepage: http://chemmethod.com
}

Original Research article

\section{Catharanthus Roseus Leaf Extract Mediated Facile Green Synthesis of Copper Oxide Nanoparticles and Its Photocatalytic Activity}

Anbuvannan Maria *, Maria Vinosel Vincenta, Ramesh Mookkaiahb, Rajesh Subramanic, Kannadasan Nadesan $^{\mathrm{d}}$

a Department of Physics, Sri Akilandeswari Women's College, Wandiwash-604408, Tamil Nadu, India

${ }^{b}$ Department of Physics, M.V. Muthiah Government Arts College for Women, Dindigul-624 001, Tamil Nadu, India

c Department of Physics, Pachaiyappa's College for Men, Kanchipuram-631501, Tamil Nadu, India

${ }^{\mathrm{d}}$ Department of Physics, E.R.K Arts and Science College, Dharmapuri-636905, Tamil Nadu, India

\section{ARTICLE INFORMATION}

Received: 28 October 2019

Received in revised: 10 December 2019

Accepted: 09 February 2020

Available online: 01 July 2020

DOI: $10.33945 /$ SAMI/CHEMM.2020.4.5

\section{KEYWORDS}

\section{CuO nanorods}

Catharanthus roseus

Rose Bengal

Photodegradation

\begin{abstract}
In this research study, the sustainable and eco-friendly green synthesis report on the use of plant extract alternate to chemicals was discussed. The Catharanthus roseus extract was used as a natural reagent to synthesize nanoparticles a brief discussion on the usage of plant extract were also summarized. Copper oxide nanoparticles (NPs) were successfully prepared using a simple way involving the combination reaction between copper nitrate and leaf extract. The synthesis under leaf extract response played an important role and led to the formation of copper oxide NPs of different size and shapes. The catalyst was characterized by XRD, FTIR, UV-Vis-DRS, PL, SEM with EDX and TEM analysis. The crystalline structure and phase identification was examined using XRD analysis, The XRD results revealed formation of pure phase without any post annealing. Formation of the metal oxygen bond of $\mathrm{CuO}$ nanoparticles was confirmed by Fourier transform infrared spectroscopy (FTIR). The surface morphology of the $\mathrm{CuO}$ nanoparticles depicted nanorods and the size difference depends upon the method of synthesis. EDX analysis confirmed the phase-purity of the as synthesized nanoparticles. UV-Vis DRS of the as obtained nanorods exhibited the absorbance in the visible region. Photocatalytic activities of the $\mathrm{CuO}$ nanoparticles were evaluated based on photodegradation of rose Bengal under UV light irradiation. The results suggested that the nanocatalyst $\mathrm{CuO}$ has potential applications as an efficient catalytic material with high efficiency for the photocatalytic degradation of organic pollutants in aqueous solution under UV light irradiation.

Copyright @ 2020 by SPC (Sami Publishing Company)

Chemical Methodologies: http://www.chemmethod.com/
\end{abstract}

*Corresponding author: E-mail: anbuphy85@gmail.com, Department of Physics, Sri Akilandeswari Women's College,

Wandiwash-604408, Tamil Nadu, India, Tel: +919843318360 


\section{Graphical Abstract}

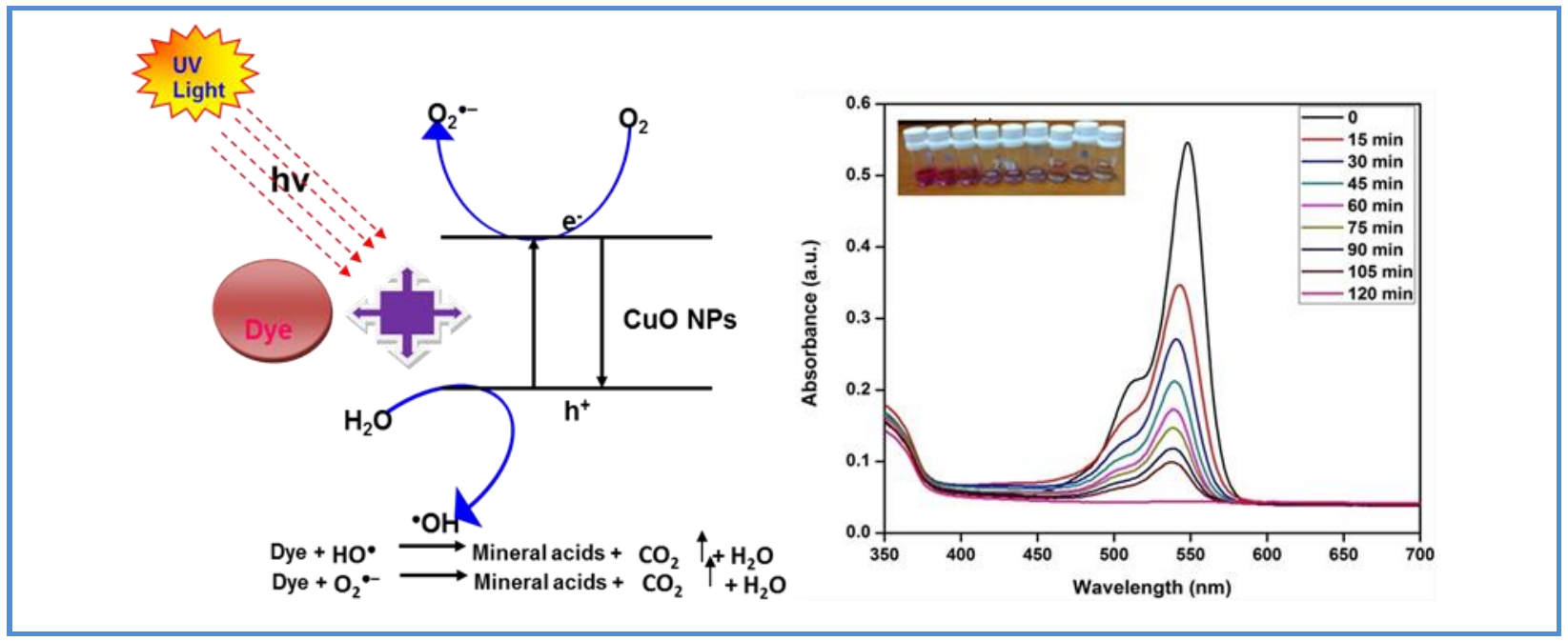

\section{Introduction}

Copper oxide $(\mathrm{CuO})$ is one of the potential $p$-type semiconductors and gains considerable attentions due to its excellent optical, electrical, physical, and magnetic properties. The possibility of manipulating bandgap of $\mathrm{CuO}$ NPs through quantum confinement from 1.2 (bulk) to $>2 \mathrm{eV}$ acts as an interesting optical property [1-8]. With altered bandgap $\mathrm{CuO}$ nanoparticles becomes highly versatile and attractive material for more range of applications. For instance it would be possible to use these nanoparticles for tunable properties as absorber or transport blocking layer in photovoltaic devices. $\mathrm{CuO}$ with narrow band gap of $1.2 \mathrm{eV}$ has been extensively used in various applications such as catalysis, solar energy conversion, gas sensor, field emission and environmental science [9]. However, these novel properties can be improved by synthesizing in $\mathrm{CuO}$ nanostructures with excellent performance comparing to that of the bulk counterpart.

Elemental copper has been identified to be harmful to humans, the environment, and aquatic life when it exceeds a particular threshold. Nevertheless, copper and its complexes have been utilized over the years by humans in water purification, pesticides as antibacterial and anti-fouling agents $[10,11]$. Different nanostructures of $\mathrm{CuO}$ are synthesized in form of nanowire, nanorod, nanoneedle, nano-flower and nanoparticle. There are various techniques opted to synthesize $\mathrm{CuO}$ nanoparticles, such as sol-gel, hydrothermal, chemical vapour deposition, co-precipitation, thermal decomposition, and combustion method [12]. Recently, there have been several techniques used to synthesize functional materials through greener approaches for achieving materials with variety of properties. Also, these methods avoid the extensive use of hazardous chemicals for the synthesis process $[13,14]$. Efforts have been taken for the application of plant extracts to fabricate different 
metal oxides and metallic nanoparticles, due to their availability, low cost and biocompatibility $[15,16]$. Catharanthus roseus one of the most medicinally valuable plant species of apocynaceae family, which is used in traditional herbal medicine and the chemical extraction has a role in cancer treatment. The leaf extract has some of the chemical constituents such as carbohydrate, flavinoid, saponin and alkaloids. Alkaloids are the most potentially active chemical constituents of Catharanthus roseus. More than 400 alkaloids are present in the plant, which are used as pharmaceuticals, agrochemicals, flavor and fragrance, ingredients, food additives and pesticides. Vincristine and vinblastine alkaloids are used in the treatment of various types of lymphoma and leukemia. All parts of the Catharanthus roseus have been used for different medical purposes such as anti-diabetic (leaves), hypolipidemic, antioxidant and Alzheimer's disease [17]. In this work, we report the green synthesis of $\mathrm{CuO}$ nanorods using Catharanthus roseus leaf extract. The as synthesized nanorods were characterized using XRD, FTIR, SEM, TEM, UV and PL respectively. The photocatalytic activity of the sample has been investigated.

\section{Experimental}

\section{Preparation of leaf extract}

The collected leaves were washed to remove the dust particles. $10 \mathrm{~g}$ of fine cut leaves were taken and mixed with $50 \mathrm{~mL}$ of deionised water. The process of boiling the mixture for 20 min changed the color of the aqueous solution from colorless to the light yellow. Allowing the mixture solution to cool to room temperature, the leaf extract was collected by filtering the mixture using Whatman filter paper.

\section{Preparation of copper oxide NPs}

Typically $\mathrm{CuO}$ nanoparticles were prepared using $20 \mathrm{~mL}$ leaf extract. Under constant stirring $2 \mathrm{gm}$ of copper nitrate were added to the above solution when the temperature attains $60^{\circ} \mathrm{C}$. Transformation of color appears in the mixture under constant heating. The dry precipitate is further transferred to the crucible and calcined at $400{ }^{\circ} \mathrm{C}$ for $4 \mathrm{~h}$. The as synthesized nanoparticles were taken for further characterization.

\section{Characterization}

The crystalline structure of the sample was characterized use of PAN analytical X'PERT PRO model XRay diffractometer. FTIR spectra were recorded under the identical conditions in the region 400-4000 $\mathrm{cm}^{-1}$ using Fourier transform infrared spectrometer (SHIMADZHU). The UV-Vis diffuse reflectance spectra (UV-Vis-DRS) recorded in UV140404B model in the wavelength range from 200-850 nm in reflectance mode. PL spectrum of the samples was recorded using FLUOROLOG-FL3-11 fluorescence 
spectrometer. The morphology of the sample was investigated by high resolution scanning electron microscopy (Quanta 200 FEG) and HR-TEM (JEM-2100) measurements revealed the morphology and size distribution.

\section{Photocatalytic activity}

The photocatalytic activity was estimated by measuring the decomposition rate of rose Bengal aqueous solutions under UV light irradiation. The UV light irradiation was carried out using a projection lamp in a photoreactor. The suspensions were prepared by adding the $200 \mathrm{mg}$ of catalyst in $500 \mathrm{~mL}$ of aqueous RB solution taken with an initial concentration of $1 \times 10^{-4} \mathrm{~mol} / \mathrm{L}$. The suspension containing $\mathrm{RB}$ and the photocatalyst was irradiated while being continuously stirred. The prepared samples from the suspension were collected at regular intervals of time, centrifuged and filtered. The degradation concentration of RB under each irradiation time of the sample was analysed using UV-visible spectrophotometer at a wavelength of $559 \mathrm{~nm}$. The photocatalytic efficiency was calculated using the expression [18].

$$
\eta=\left(1-\mathrm{C} / \mathrm{C}_{0}\right) * 100
$$

Where $\mathrm{C}_{0}$ is the concentration of $\mathrm{RB}$ before illumination and $\mathrm{C}$ is the concentration after irradiation time.

\section{Results and discussion}

\section{XRD analysis}

The X-ray diffraction pattern of CuO nanoparticles were obtained using a Phillip PW 1800 X-ray diffractometer with $\mathrm{Cu} \mathrm{K} \alpha$ radiation of wavelength $1.5405 \AA$ operated at $40 \mathrm{KV}$ are shown in the Figure 1. The prominent diffraction peak values at $2 \theta=32.44^{\circ}, 35.52^{\circ}, 38.66^{\circ}, 48.64^{\circ}, 53.37^{\circ}$,

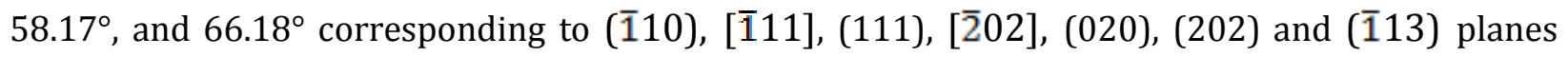
respectively. The configured diffraction peaks can be indexed to pure monoclinic structure crystalline phase with the lattice parameters $a=4.636 \AA, b=3.43 \AA$ and $c=5.136 \AA$, which are close enough to the standard values (JCPDS No: 80-1916). No other impurity peaks were spotted. The pure crystalline phase of $\mathrm{CuO}$ nanoparticles with small crystallite size were put forward by broad diffraction peaks. The mean crystallite sizes (D) of the $\mathrm{CuO}$ nanoparticles were calculated using Debye Scherrer's formula [19].

$$
\mathrm{D}=\frac{\mathrm{K} \lambda}{\beta \operatorname{Cos} \theta} \AA
$$


Where $d$ is the average crystallite size $(\mathrm{nm}), \mathrm{K}$ is the grain shape factor (0.9), $\lambda$ is the X-ray wavelength $(\mathrm{nm}), \beta$ is the line broadening at half the maximum intensity in radians, and $\theta$ is the Bragg diffraction angle of the $2 \theta$ peak. The average crystallite size of $\mathrm{CuO}$ nanoparticles was found to be $23 \mathrm{~nm}$.

\section{Optical studies}

Figure 2a. exhibits the optical absorption spectrum of CuO NPs synthesized by Catharanthus roseus extract. The absorption spectra were used to study the energy band and the type of electronic transitions, which shows a strong fundamental absorption edge approximately at $700 \mathrm{~nm}$ due to direct transition of electrons [20]. The optical absorption spectra value can be calculated using the equation [21].

$$
\mathrm{E}_{\mathrm{g}}=\frac{h_{c}}{\lambda} \mathrm{eV} ; \mathrm{E}_{\mathrm{g}}=\frac{1240}{\lambda} \mathrm{eV}
$$

Where, $\mathrm{E}_{\mathrm{g}}$ is the band gap energy $(\mathrm{eV}), \mathrm{h}$ is the Planck's constant $\left(6.626 \times 10^{-34} \mathrm{Js}\right), \mathrm{C}$ is the light velocity $\left(3 \times 10^{8} \mathrm{~m} / \mathrm{s}\right)$ and $\lambda$ is the wavelength $(\mathrm{nm})$. From Figure $1 \mathrm{a}$, the absorption edge is positioned at $700 \mathrm{~nm}$, which corresponding to the band gap values of $1.79 \mathrm{eV}$. The reflectance spectra were analyzed using the Kubelka-Munk relation (equation 3). Equation 3 was utilized to convert the reflectance data into a Kubelka-Munk function (equivalent to the absorption coefficient) F (R).

$$
F(R)=\frac{(1-R)^{2}}{2 R}
$$

Where, $\mathrm{R}$ is the reflectance value. Figure $2 \mathrm{~b}$. illustrates the Kubelka-Munk plots for the CuO NPs. It is used to determine the band gap energy associated with indirect transitions. The CuO NPs exhibits indirect $\mathrm{E}_{\mathrm{g}}$ value of $1.72 \mathrm{eV}$. The $\mathrm{Eg}$ can be obtained by extrapolating the linear portion to the photon energy axis. The extrapolated value of direct and indirect bandgap of $\mathrm{CuO}$ nanoparticles were $1.79 \mathrm{eV}$ and $1.72 \mathrm{eV}$ respectively, which is much larger than the bulk material. When the radius of the $\mathrm{CuO}$ nanoparticles approaches the exciton-Bohr radius the widening of the band gap is observed. If the direct band gap is higher than the indirect band gap, the materials will be crystalline. The observed increasing band gap could be ascribed by the presence of intragap states and quantum confinement effect [22]. 


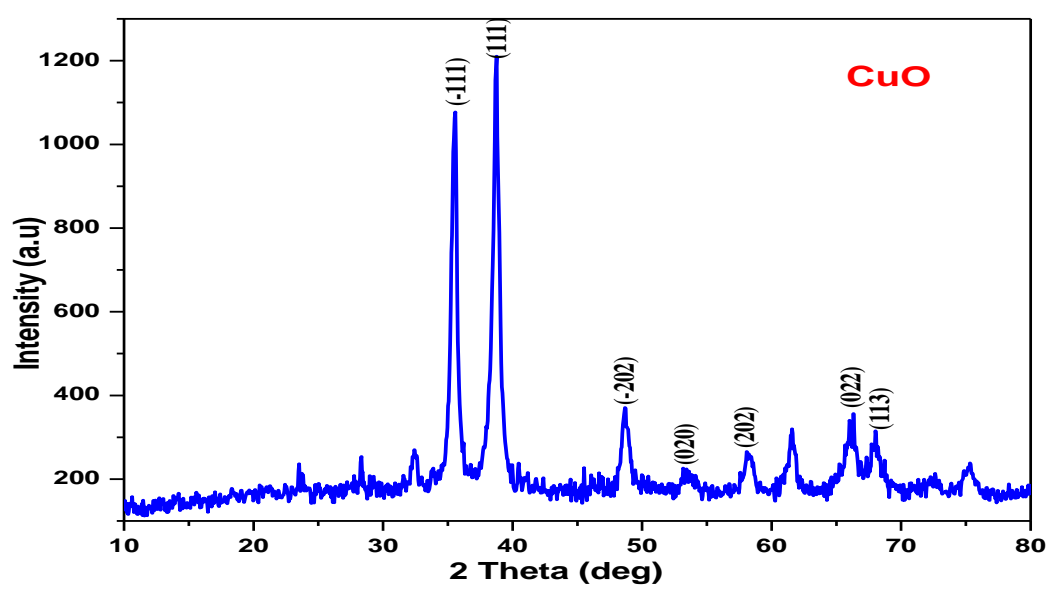

Figure1. XRD pattern of $\mathrm{CuO}$ nanoparticles

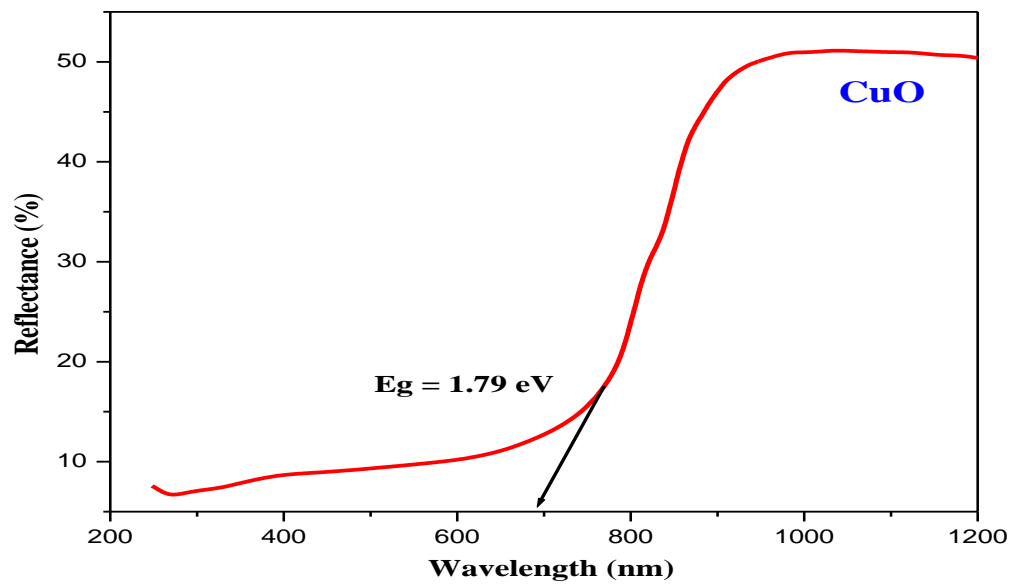

Figure 2a. UV-DRS spectrum of $\mathrm{CuO}$ nanoparticles

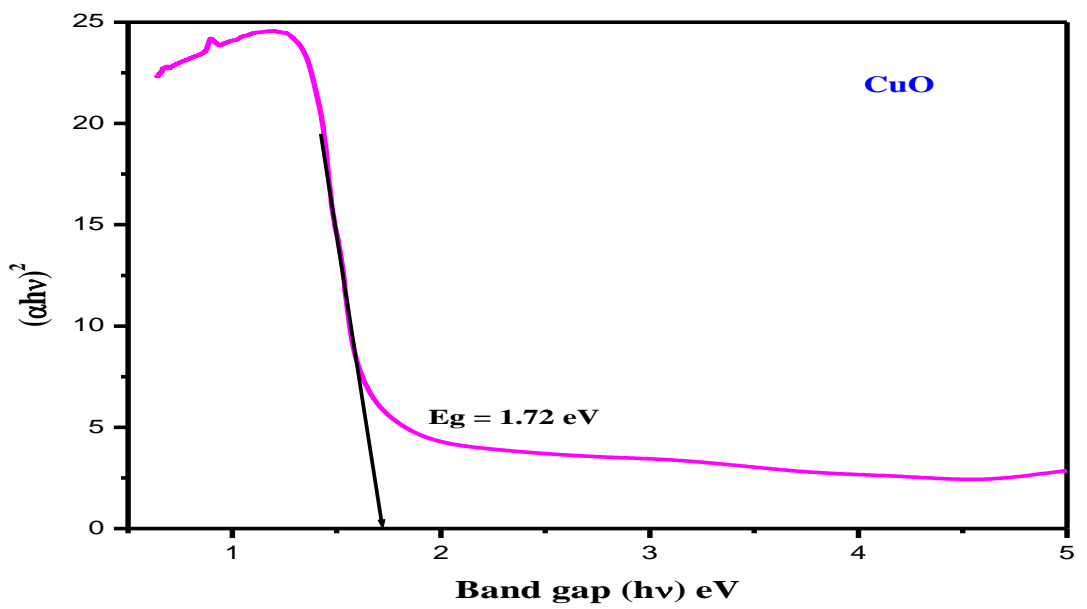

Figure 2b. Plot of indirect band gap of $\mathrm{CuO}$ nanoparticles 


\section{PL analysis}

The room temperature PL spectrum of $\mathrm{CuO}$ nanoparticles is an effective method to evaluate defect characteristics and optical properties of the sample prepared by Catharanthus roseus were shown in Figure 3. In PL analysis two emission peaks are observed at $410 \mathrm{~nm}$ (violet), 450 $\mathrm{nm}$ and $468 \mathrm{~nm}$ (blue) for $\mathrm{CuO}$ nanoparticles. The first one corresponds to the band-edge emission and second one is due to artifact [23].

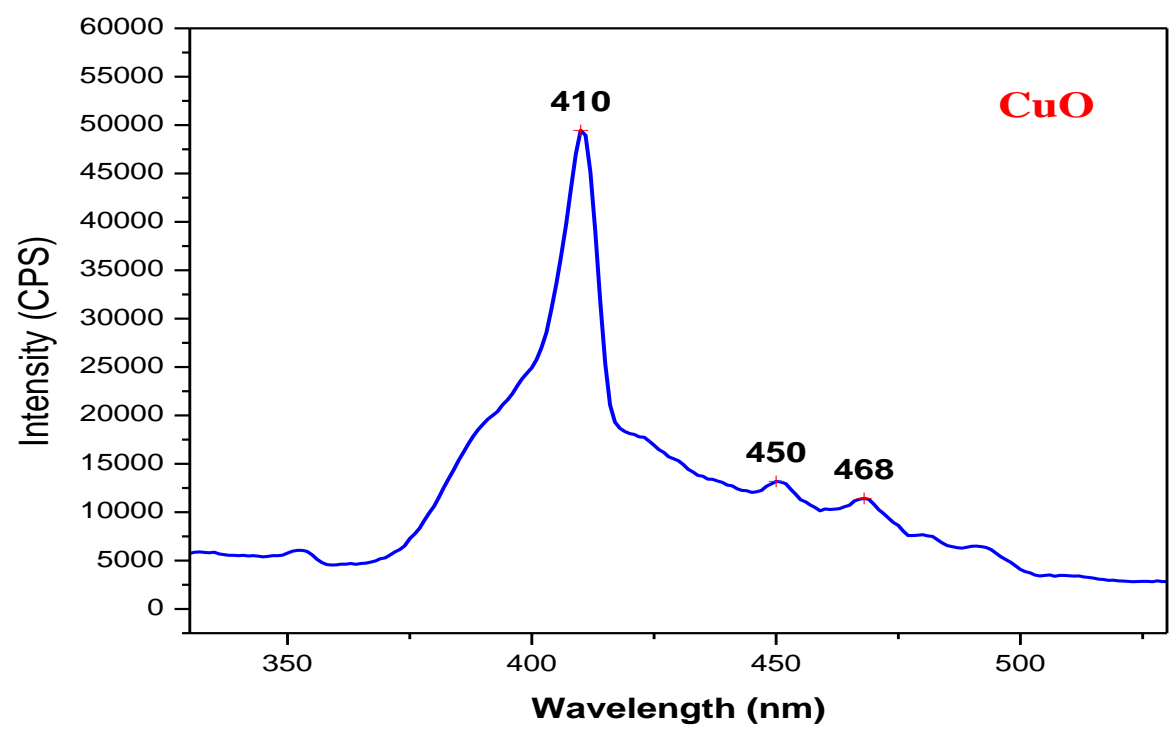

Figure 3. PL spectrum of as synthesized $\mathrm{CuO}$ nanoparticles

\section{FT-IR analysis}

The FTIR transmission spectral analysis of $\mathrm{CuO}$ nanoparticles are depicted in Figure 4. FT-IR analysis was used to determine the functional groups of copper oxide nanoparticles. The intense bands at $520 \mathrm{~cm}^{-1}$ were assigned to be symmetric and asymmetric stretching modes of $\mathrm{Cu}-\mathrm{O}$ metal oxygen bond [24]. The broad absorption peaks at $3443 \mathrm{~cm}^{-1}$ are due to the presence of $\mathrm{O}-\mathrm{H}$ stretching and bending vibrations of hydroxyl group, which is probably due to the fact that the spectra were not recorded in in-situ and some re-adsorption of water from the ambient atmosphere, occurred [25]. The existence of prominent IR bands ranging at 400-600 $\mathrm{cm}^{-1}$ confirmed the formation of $\mathrm{CuO}$ nanoparticles from the green synthesis. It is prominent that no infrared active modes from $\mathrm{Cu}_{2} \mathrm{O}$ were detected, which is in good agreement with the XRD result. 


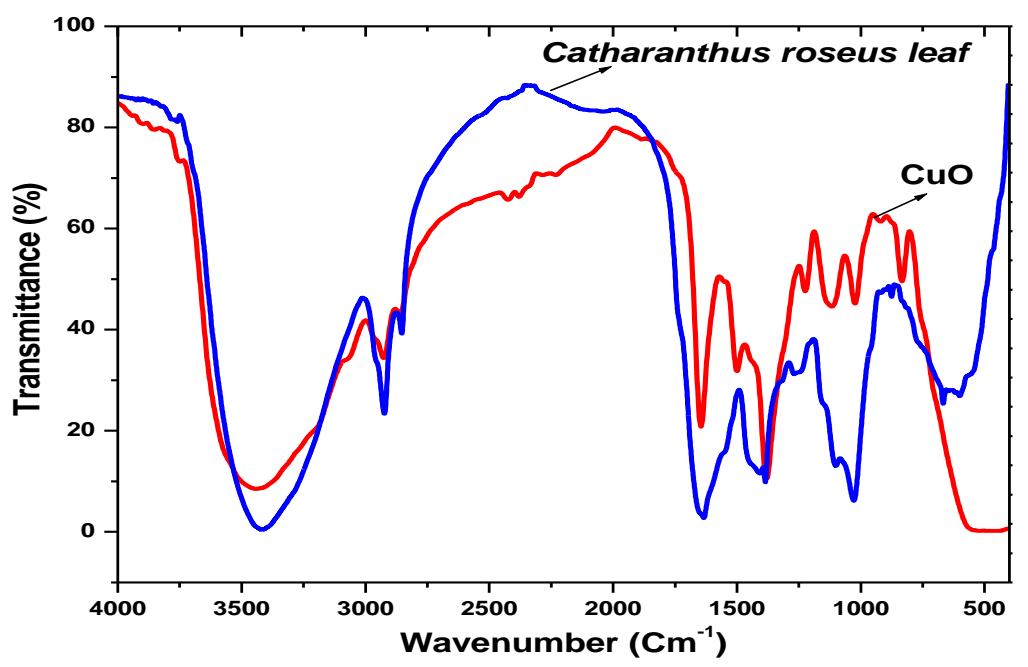

Figure 4. FT-IR spectra of leaf extract and $\mathrm{CuO}$ nanoparticles

\section{SEM analysis}

Surface morphology and elemental composition of the copper oxide nanoparticles were probed using the high resolution scanning electron microscope. The structure of $\mathrm{CuO}$ nanoparticles for different magnification is shown in Figure 5a. It was observed that $\mathrm{CuO}$ nanoparticles resembled nanorod like morphology with homogenous distribution and slight agglomeration. The elemental analysis of the sample was performed using energy dispersive X-ray spectroscopy (EDX). The EDX spectra of the CuO nanoparticles are depicted in Figure $5 \mathrm{~b}$. This confirms the stochiometric proportion of $\mathrm{Cu}$ and $\mathrm{O}$ elements in the sample.
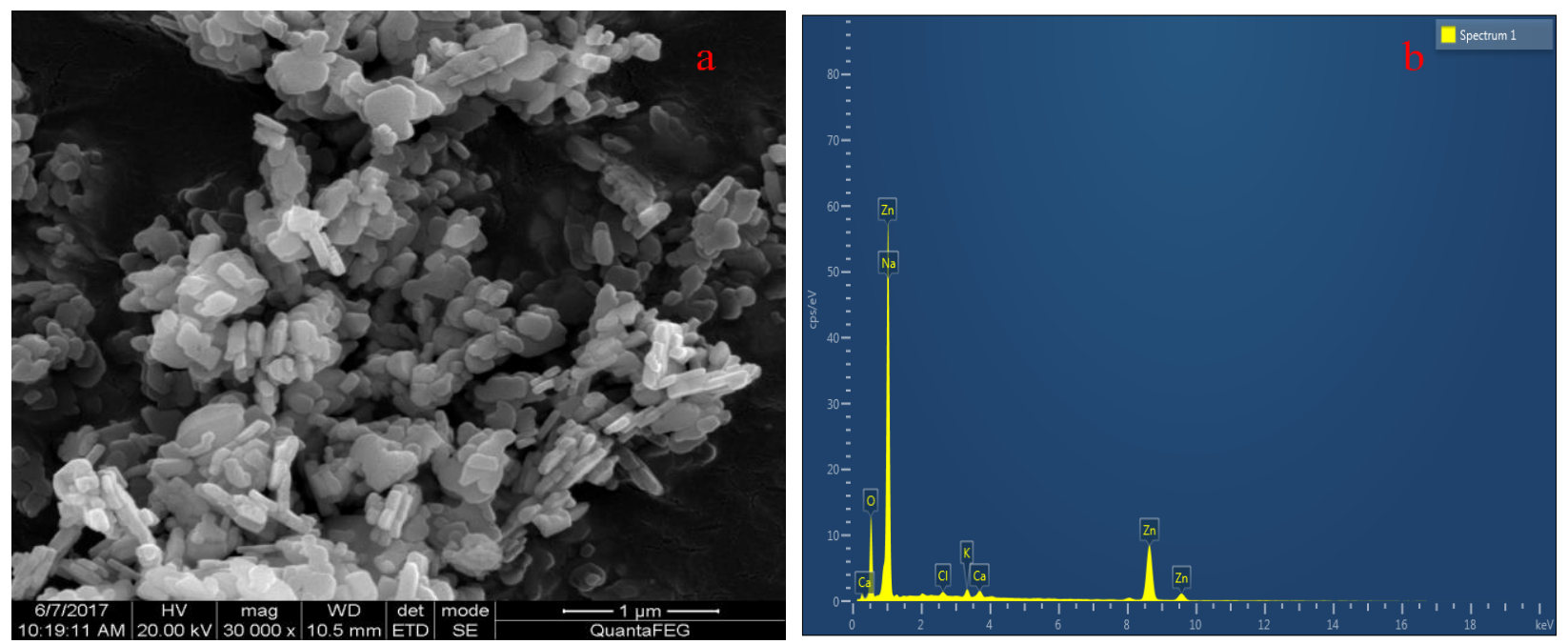

Figure 5. HR-SEM micrograph and EDX spectrum of $\mathrm{CuO}$ nanoparticles synthesized using Catharanthus roseus leaf extract 


\section{TEM analysis}

Microstructure of the $\mathrm{CuO}$ nanoparticles was analyzed using the high resolution transmission electron microscope (HR-TEM). Figure 6a-b. demonstrates the HR-TEM and selected area of electron diffraction (SAED) pattern of the $\mathrm{CuO}$ nanoparticles prepared using the green synthesis method. It exhibits nanorod like structure and its size observed to be $23 \mathrm{~nm}$. Diffraction rings of the SAED pattern matches well with the prominent (hkl) values of XRD pattern. It was found that the particles were well crystallized. This proves the monoclinic structure of the $\mathrm{CuO}$ nanoparticles.

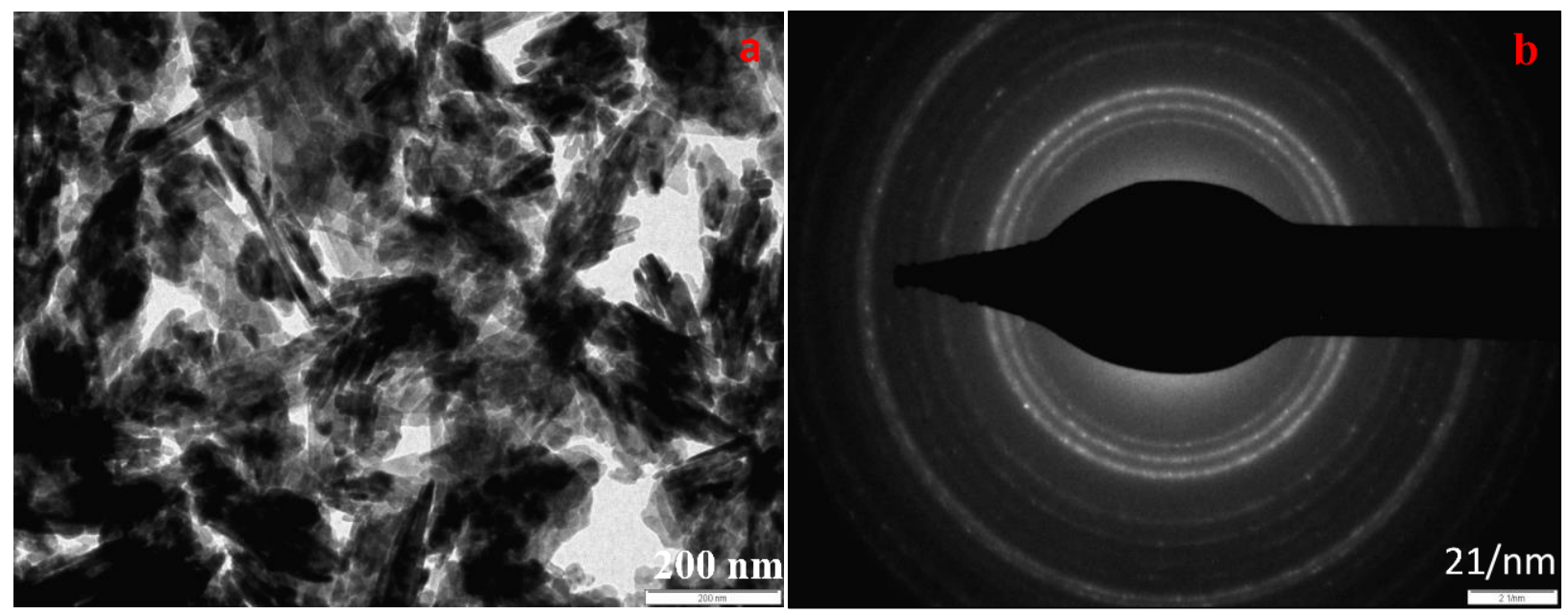

Figure 6. HR-TEM image and SAED pattern of CuO nanoparticles

\section{Photocatalytic activity}

Photodegradation of rose Bengal was performed using a catalyst concentration of $200 \mathrm{mg} / 500 \mathrm{~mL}$, neutral $\mathrm{pH}$, and an irradiation time of $120 \mathrm{~min}$ over $\mathrm{CuO}$ nanoparticles. It was witnessed that the photodegradation efficiency of rose Bengal was inversely proportional to its concentration, which means, the lower is the dye concentration, the higher efficiency of the dye photodegradation at fixed concentrations of catalyst. The photodegradation efficiency depends on the formation of hydroxyl radicals, which is the critical species in the degradation process. Due to static concentration of the catalyst there are only fewer active sites for adsorption of HO- so the generation of HO- will be reduced [26]. On irradiation with UV light, the maximum degradation was obtained using CuO nanoparticles (86\%), as shown in Figure 7. The reason could be that $\mathrm{CuO}$ nanoparticles have more surface area and greater pore size. The enhanced catalytic behavior of the $\mathrm{CuO}$ can be established from the facts of increased surface area, rod-like morphology, low charge transfer resistance and reduced band gap. The photocatalytic activity majorly depends on generation of charge carriers, transfer of charge carriers, and chemical reaction process. On photoexcitation, the charge carrier electrons and holes are generated and 
these generated charge carriers may migrate to the surface of the catalysts and react with the adsorbents in the desired process. The electrons then react with and absorb oxygen molecules to form superoxide radicals, which are responsible for the degradation of the organic pollutant $[27,28]$. The enhanced $\mathrm{PCD}$ achieved using $\mathrm{CuO}$ is attributed to a lower recombination rate of the electron-hole pairs in $\mathrm{CuO}$, as a result of efficient interparticle electron transfer. It can also be attributed to the better crystallinity of the sample. The changes in the absorption spectra of the rose Bengal exposed to UV light for various irradiation times $(0,15,30,45,60,75,90,105$, and $120 \mathrm{~min})$ at the presence of $\mathrm{CuO}$ nanoparticles. The absorption maxima at $559 \mathrm{~nm}$ decreased gradually with irradiation time.

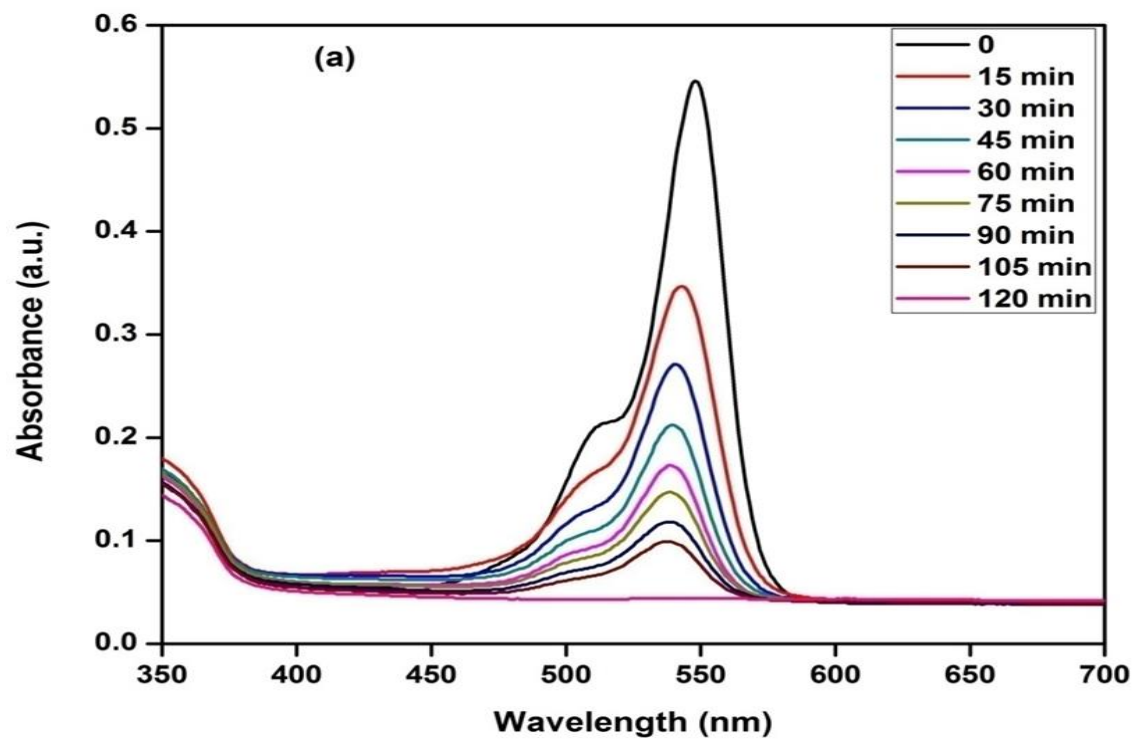

Figure 7. Photocatalytic degradation of rose Bengal under UV light irradiation of CuO nanoparticles

\section{Probable mechanism for photodegradation of rose Bengal (RB) using synthesized CuO NPs}

On illuminating the nanocatalyst surface (CuO NPs) with light energy higher than its band-gap energy, leads to the formation of holes $\left(\mathrm{h}^{+}\right)$in the valence band and an electron $\left(\mathrm{e}^{-}\right)$in the conduction band of $\mathrm{CuO}$ nanoparticles. The holes $\left(\mathrm{h}^{+}\right)$act as an oxidizing agent and oxidize the pollutant directly or react with water to form hydroxyl radicals. The electron $\left(\mathrm{e}^{-}\right)$in the conduction band acts as a reducing agent and reduces the oxygen adsorbed on the surface of $\mathrm{CuO}$ photocatalyst.

The plausible mechanism for the photocatalytic degradation of RB dye can be schematically shown as Photocatalysts $(\mathrm{CuO})+\mathrm{h} v \rightarrow \mathrm{e}^{-}+\mathrm{h}^{+}$

$$
\begin{gathered}
\mathrm{h}^{+}+\mathrm{H}_{2} \mathrm{O} \rightarrow \mathrm{H}^{+}+\mathrm{OH}^{\bullet} \\
\mathrm{h}^{+}+\mathrm{OH}^{-} \rightarrow \mathrm{OH}^{\bullet}
\end{gathered}
$$




$$
\begin{gathered}
\mathrm{e}^{-}+\mathrm{O}_{2} \rightarrow \mathrm{O}_{2}^{-} \\
2 \mathrm{e}^{-}+\mathrm{O}_{2}+2 \mathrm{H}^{+} \rightarrow \mathrm{H}_{2} \mathrm{O}_{2} \\
\mathrm{e}^{-}+\mathrm{H}_{2} \mathrm{O}_{2} \rightarrow \mathrm{OH}^{\bullet}+\mathrm{OH}^{-}
\end{gathered}
$$

Organic dye $+\cdot \cdot \mathrm{OH}+\mathrm{O}_{2} \rightarrow \mathrm{CO}_{2}+\mathrm{H}_{2} \mathrm{O}+$ other degradation productions

The excited dye injects an electron to the conduction band of $\mathrm{CuO}$, and scavenged by pre-adsorbed oxygen $\mathrm{O}_{2}$, to form active oxygen radicals. These generated active radicals drive the photodegradation process. The as prepared $\mathrm{CuO}$ nanoparticles play an important role as an electron carrier. Such assisted photo processes provide an attractive path for the treatment of dyes under sunlight (See Scheme 1).

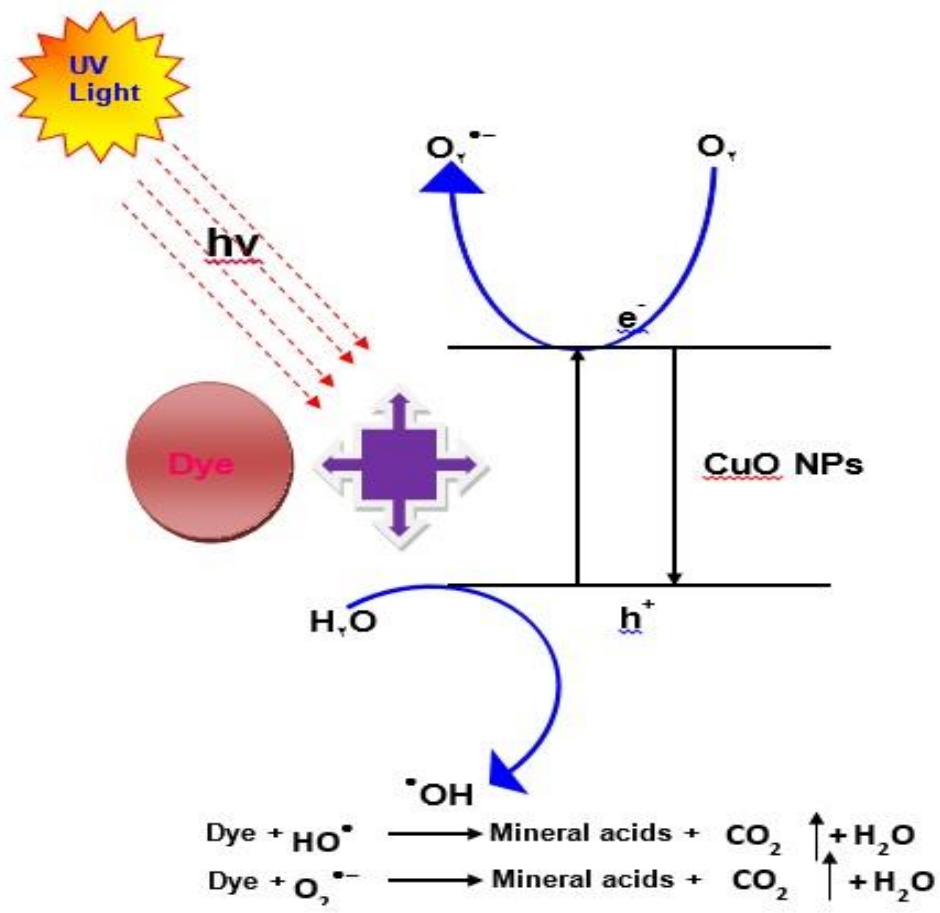

Scheme 1. Schematic representation of photodegradation mechanism of $\mathrm{CuO}$ nanoparticles

\section{Conclusions}

$\mathrm{CuO}$ NPs were successfully synthesized using the leaf mediated green method with the assistance of the concentration of Catharanthus roseus leaves extract. The synthesis method could be a convenient route to further processing or applications of size (20-30 nm) rod like structure nanoparticles. Catharanthus roseus leaf extract helps to control particle size and morphology of the products. The XRD analysis demonstrated that the NPs were crystalline with a monoclinic 
structure. Photocatalytic degradation under light irradiation acts as a promising application in the development of new combination semiconductor metal oxide nanocomposites. Further antibacterial and microbial activity of the sample can also be studied for the waste water management.

\section{Acknowledgements}

The authors are thankful and grateful to Department of Physics, Sri Akilandeswari Women's College, Wandiwash, Tamil Nadu, India-604 408 for providing all necessary facilities to carry out the present work successfully.

\section{Conflict of Interest}

We have no conflicts of interest to disclose.

\section{References}

[1] Chauhan M., Sharma B., Kumar R., Chaudhary G.R., Hassan A.A., Kumar S. Environ Res., 2019, 168:85

[2] Gebremedhn K., Kahsay M.H., Aklilu M. J. Phar. Pharmacol., 2019, 7:327

[3] Wang W., Wang L., Shi H., Liang Y. CrystEngComm., 2012, 14:5914

[4] Yang C., Xiao F., Wang J., Su X. J. Coll. Interface Sci., 2014, 34:435

[5] Borgohain K., Singh J.B., Rama Rao M.V., Shripathi T., Mahamuni S. Phys. Rev. B, 2000, 61:11093

[6] Xu Y., Chen D., Jiao X. J. Phys. Chem. B, 2005, 109:13561

[7] Meshram S.P., Adhyapak P.V., Mulik U.P., Amalnerkar D.P. Chem. Eng. J., 2012, 204:158

[8] Langmar O., Ganivet C.R., Lennert A., Costa R.D., de laTorre G., Torres T., Guldi D.M. Angew. Chem. Int. Ed. Engl., 2015, 54:7688

[9] Chandan T., Indranirekha S., Moushumi H., Manash R.D. RSC Adv., 2014, 4:53229

[10] Padil V.V.T., Černík M. Int. J. Nanomedicine., 2013, 8:889

[11] Nagajyothi P.C., Muthuraman P., Sreekanth T.V.M., Kim D.H., Shim J. Arab. J. Chem., 2017, 10:215

[12] Vinosel V.M., Amaliya, A.P., Blessi, S. and Pauline, S., Inter. Res. J. Eng. Tech., 2017, 4:106

[13] Ye T., Guiwen Z., Weiping Z., Shangda X. Mater. Res. Bull., 1997, 32:501

[14] Suresh D., Nethravathi P.C., Udayabhanu, Rajanaika H., Nagabhushana H., Sharma S.C. Mater. Sci. Semicond. Process., 2015, 31:446

[15] Zhang D., Yang D.C. Trends Biotechnol., 2016, 34:588

[16] Ahmed S., Ahmad M., Swami B.L., Ikram S., J. Adv. Res., 2016, 7:17

[17] Sharma K., Choudhary A.S., Chauhan E.S. World J. Pharm. Res., 2018, 9:1281

[18] Maria Vinosel V., Anand S., Asisi Janifer M., Pauline S. Mater. Today Proceed., 2019, 8:301 
[19] Anbuvannan M., Ramesh M., Viruthagiri G., Shanmugam N., Kannadasan N., Mat. Sci. Semicon. Process., 2015, 39:621

[20] Dhineshbabu N.R., Rajendran V., Nithyavathy N., Vetumperumal R. Appl. Nanosci., 2016, 6:933

[21] Anbuvannan M., Ramesh M, Viruthagiri G., Shanmugam N., Kannadasan N. Spectrochim. Acta Part A Mol. Biomol. Spectroscopy, 2015, 143:304

[22] Velusamy T., Liguori A., Macias-Montero M., Babu Padmanaban D., Carolan D., Gherardi M., Colombo V., Maguire P., Svrcek V., Mariotti D. Plasma Pro. Polym., 2017, 14:e1600224

[23] Ningthoujam R.S., Sudarsan V., Kulshreshtha S.K. J. Lumines., 2007, 127:747

[24] Rayapa Reddy K. J. Mol. Struct., 2017, 1150:553

[25] Mari A., Mookkaiah R., Elayaperumal M. Asian J. Green Chem., 2019, 3:418

[26] Sherly E.D., Vijaya J. J., Selvam N. C. S., Kennedy L. J., Cer. Inter., 2014, 40:5681

[27] Kayaci F., Vempati S., Ozgit-Akgun C., Donmez I., Biyikli N., Uyar T., Nanoscale, 2014, 6:5735

[28] Anbuvannan M., Ramesh M., Manikandan E., Srinivasan R. Asian J. Nanosci. Mater., 2019, 2:99

How to cite this manuscript: Anbuvannan Mari*, Maria Vinosel Vincent, Ramesh Mookkaiah, Rajesh Subramani, Kannadasan Nadesan, Catharanthus Roseus Leaf Extract Mediated Facile Green Synthesis of Copper Oxide Nanoparticles and Its Photocatalytic Activity. Chemical Methodologies 4(4), 2020, 424-436. DOI:10.33945/SAMI/CHEMM.2020.4.5. 